\title{
Flag-Transitive 6-(v, k, 2) Designs
}

\author{
Xiaolian Liao', Shangzhao Li $^{2}$, Guohua Chen ${ }^{1}$ \\ ${ }^{1}$ Department of Mathematics, Hunan University of Humanities Science and Technology, Loudi, China \\ ${ }^{2}$ Department of Mathematics, Changshu Institute of Technology, Changshu, China \\ Email: hnldlxl2005@126.com
}

Received 28 February 2014; revised 28 March 2014; accepted 15 April 2014

Copyright (C) 2014 by authors and Scientific Research Publishing Inc.

This work is licensed under the Creative Commons Attribution International License (CC BY).

http://creativecommons.org/licenses/by/4.0/

cC) (7) Open Access

\begin{abstract}
The automorphism group of a flag-transitive $6-(v, k, 2)$ design is a 3-homogeneous permutation group. Therefore, using the classification theorem of 3-homogeneous permutation groups, the classification of flag-transitive $6-(\mathrm{v}, \mathrm{k}, 2)$ designs can be discussed. In this paper, by analyzing the combination quantity relation of $6-(v, k, 2)$ design and the characteristics of 3-homogeneous permutation groups, it is proved that: there are no $6-(\mathrm{v}, \mathrm{k}, 2)$ designs $\mathrm{D}$ admitting a flag transitive group $G \leq$ Aut (D) of automorphisms.
\end{abstract}

\section{Keywords}

Flag-Transitive, Combinatorial Design, Permutation Group, Affine Group, 3-Homogeneous Permutation Groups

\section{Introduction}

For positive integers $t \leq k \leq v$ and $\lambda$, we define a $t-(v, k, \lambda)$ design to be a finite incidence structure $D=(X, \mathrm{~B}, I)$, where $X$ denotes a set of points, $|X|=v$ and $\mathrm{B}$ a set of blocks, $|\mathrm{B}|=b$, with the properties that each block $B \in \mathrm{B}$ is incident with $k$ points, and each $t$-subset of $X$ is incident with $\lambda$ blocks. A flag of $D$ is an incident point-block pair, that is $x \in X$ and $B \in \mathrm{B}$ such that $(x, B) \in I$. We consider automorphisms of $D$ as pairs of permutations on $X$ and B which preserve incidence, and call a group $G \leq \operatorname{Aut}(D)$ of automorphisms of $D$ flag-transitive (respectively block-transitive, point t-transitive, point t-homogeneous), if $\mathrm{G}$ acts transitively on the flags (respectively transitively on the blocks, t-transitively on the points, $t$-homogeneous on the points) of $D$. It is a different problem in Combinatorial Maths how to construct a design with given parameters. In this paper, we shall take use of the automorphism groups of designs to find some new designs.

In recent years, the classification of flag-transitive Steiner 2-designs has been completed by W. M. Kantor (See [1]), F. Buekenhout, A. De-landtsheer, J. Doyen, P. B. Kleidman, M. W. Liebeck, J. Sax (See [2]); for flag- 
transitive Steiner $t$-designs $(2<t \leq 6)$, Michael Huber has done the classification (See [3]-[7]). But only a few people have discussed the case of flag-transitive t-designs where $t>3$ and $\lambda>1$.

In this paper, we may study a kind of flag-transitive designs with $\lambda=2$. We may consider this problem by making use of the classification of the finite 3-homogeneous permutation groups to study flag-transitive $6-(v, k, 2)$ designs. Our main result is:

Theorem: There are no non-trivial $6-(v, k, 2)$ designs $D$ admitting a flag transitive group $G \leq \operatorname{Aut}(D)$ of automorphisms.

\section{Preliminary Results}

Lemma 2.1. (Huber $M[4])$ Let $D=(X, B, I)$ be a $t$-design with $t \geq 3$.If $G \leq \operatorname{Aut}(D)$ acts flag-transitively on $D$, then $\mathrm{G}$ also acts point 2-transitively on $D$.

Lemma 2.2. (Cameron and Praeger [8]). Let $D=(X, B, I)$ be a $t-(v, k, \lambda)$ design with $\lambda \geq 2$. Then the following holds:

(1) If $G \leq \operatorname{Aut}(D)$ acts block-transitively on $D$, then $G$ also acts point $\lfloor t / 2\rfloor$-homogeneously on $D$;

(2) If $G \leq \operatorname{Aut}(D)$ acts flag-transitively on $D$, then $G$ also acts point $\lfloor(t+1) / 2\rfloor$-homogeneously on D.

Lemma 2.3. (Huber $M[9])$ Let $D=(X, B, I)$ be a $t-(v, k, \lambda)$ design. If $G \leq \operatorname{Aut}(D)$ acts flag-transitively on $D$, then , for any $x \in X$, the division property $r|| G_{x} \mid$ holds.

Lemma 2.4. Let $D=(X, B, I)$ be a $t-(v, k, \lambda)$ design. Then the following holds:

(1) $b k=v r$;

(2) $\left(\begin{array}{l}v \\ t\end{array}\right) \lambda=b\left(\begin{array}{l}k \\ t\end{array}\right)$;

(3) For $1 \leq s<t$, a $t-(v, k, \lambda)$ design is also an $s-\left(v, k, \lambda_{s}\right)$ design, where $\lambda_{s}=\lambda \frac{\left(\begin{array}{l}v-s \\ t-s\end{array}\right)}{\left(\begin{array}{l}k-s \\ t-s\end{array}\right)}$.
(4) particular, if $t=6$, then
\[ r(k-1)(k-2)(k-3)(k-4)(k-5)=\lambda(v-1)(v-2)(v-3)(v-4)(v-5) \text {. } \]

Lemma 2.5. (Beth T [10]) If $D=(X, B, I)$ is a non-trivial $t-(v, k, \lambda)$ design, then $v>k+t$

Lemma 2.6. (Wei J L [11]) If $D=(X, B, I)$ is a $t-(v, k, \lambda)$ design, then

$$
\lambda(v-t+1) \geq(k-t+1)(k-t+2), t>2 .
$$

In this case, when $t=6$, we deduce from Lemma 2.6 the following upper bound for the positive integer $k$.

Corollary 2.7. Let $D=(X, B, I)$ be a non-trivial $6-(v, k, 2)$ design, then

$$
k \leq\left\lfloor\sqrt{2 v-\frac{39}{4}}+\frac{9}{2}\right\rfloor .
$$

Proof: By Lemma 2.6, when $t=6, \lambda=2$, we have $2(v-5) \geq(k-5)(k-4)$, then

$$
k \leq\left\lfloor\sqrt{2 v-\frac{39}{4}}+\frac{9}{2}\right\rfloor .
$$

Remark 2.8. Let $D=(X, B, I)$ be a non-trivial $t-(v, k, \lambda)$ design with $t \geq 6$. If $G \leq \operatorname{Aut}(D)$ acts flagtransitively on $D$, then by Lemma $2.2(1), G$ acts point 3-homogeneously and in particular point 2-transitively on $D$. Applying Lemma 2.4 (2) yields the equation

$$
b=\frac{\lambda\left(\begin{array}{l}
v \\
t
\end{array}\right)}{\left(\begin{array}{l}
k \\
t
\end{array}\right)}=\frac{v(v-1)\left|G_{x y}\right|}{\left|G_{B 1}\right|}
$$

where $x$ and $y$ are two distinct points in $X$ and $B_{1}$ is a block in $B$. If $x \in B_{1}$, then 


$$
2\left(\begin{array}{c}
v-2 \\
4
\end{array}\right)=(k-1)\left(\begin{array}{c}
k-2 \\
4
\end{array}\right) \frac{\left|G_{x y}\right|}{\left|G_{x B 1}\right|} .
$$

Corollary 2.9 Let $D=(X, B, I)$ be a $t-(v, k, \lambda)$ design, then

$$
\lambda\left(\begin{array}{c}
v-s \\
t-s
\end{array}\right) \equiv 0\left(\bmod \left(\begin{array}{l}
k-s \\
t-s
\end{array}\right)\right) .
$$

For each positive integers, $s \leq t$.

Let $G$ be a finite 3-homogeneous permutation group on a set $X$ with $|X| \geq 4$. Then $G$ is either of

\section{(A) Affine Type:}

$G$ contains a regular normal subgroup $T$ which is elementary Abelian of order $v=2^{d}$.If we identify $G$ with a group of affine transformations

$$
x \mapsto x^{\varepsilon}+\mu
$$

Of $V=V(d, 2)$, where $\varepsilon \in G_{0}$ and $\mu \in V$, then particularly one of the following occurs:

(1) $G \cong A G L(1,8), A \Gamma L(1,8)$ or $A \Gamma L(1,32)$;

(2) $G \cong S L(d, 2), d \geq 2$;

(3) $G \cong A_{7}, v=2^{4}$;

or

(B) Almost Simple Type: $G$ contains a simple normal subgroup $N$, and $N \leq G \leq \operatorname{Aut}(D)$. In particular, one of the following holds, where $N$ and $v=|X|$ are given as follows:

(1) $A_{v}, v \geq 5$;

(2) $\operatorname{PLS}(2, q), v=q+1, q>3$;

(3) $M_{v}, v=11,12,22,23,24$

(4) $M_{11}, v=12$.

\section{Proof of the Main Theorem}

Let $D=(X, B, I)$ be a non-trivial $6-(v, k, 2)$ design, $G \leq \operatorname{Aut}(D)$ acts flag-transitively on $D$, by lemma 2.2, $G$ is a finite 3-homogeneous permutation group. For $D$ is a non-trivial $6-(v, k, 2)$ design, then $k>6$. We will prove by contradiction that $G \leq \operatorname{Aut}(D)$ cannot act flag-transitively on any non-trivial $6-(v, k, 2)$ design.

\subsection{Groups of Automorphisms of Affine Type}

Case (1): $G \cong A G L(1,8), A \Gamma L(1,8)$ or $A \Gamma L(1,32)$;

If $v=8$, then Lemma 2.5 yields $k<v-t=2$, a contradiction to $k>6$. For $v=32$, Corollary 2.7 implies $k \leq 12$. Thus $k=7,8,9,10,11,12$. By Lemma 2.4 we have

$$
r(k-1)(k-2)(k-3)(k-4)(k-5)=2 \times 31 \times 30 \times 29 \times 28 \times 27
$$

for each values of $k$, we have

$$
r=31 \times 29 \times 7 \times 9,31 \times 29 \times 18, \frac{31 \times 29 \times 27}{4}, 31 \times 29 \times 3, \frac{31 \times 29 \times 3}{2}, \frac{31 \times 29 \times 9,}{11}
$$

but $r$ is a positive integer, thus $r=31 \times 29 \times 7 \times 9,31 \times 29 \times 18,31 \times 29 \times 3$. On the other hand, we have $\left|G_{x}\right|=5(v-1)=5 \times 31$, those are contradicting to Lemma 2.3 .

Case (2): $G \cong S L(d, 2), d \geq 2$.

Here $v=2^{d}>k>6$. For $d=3$, we have $v=8$, already ruled out in Case (1). So we may assume that $d>3$. Any six distinct points being non-coplanar in $A G(d, 2)$, they generate an affine subspace of dimension at least 3. Let $\varepsilon$ be the 3-dimensional vector subspace spanned by the first three basis vectors $e_{1}, e_{2}, e_{3}$ of the vector space $V=V(d, 2)$. Then the point-wise stabilizer of $\varepsilon$ in $S L(d, 2)$ (and therefore also in $G$ ) acts point-transitively on $V \backslash \varepsilon$. Let $B_{1}$ and $B^{\prime}$ be the two blocks which are incident with the 6-subset $\left\{0, e_{1}, e_{2}, e_{3}, e_{1}+e_{2}, e_{2}+e_{3}\right\}$, If the block $B_{1} \cup B^{\prime}$ contains some point $\alpha$ of $V \backslash \varepsilon$, then $B_{1} \cup B^{\prime}$ contains all 
points of $V \backslash \varepsilon$, and so $2 k-12 \geq v-8=2^{d}-8$, this yields $k>2^{d-1}+2>2^{d-1}+1$, a contradiction to Lemma 2.6. Hence $B_{1} \subseteq \varepsilon$ and $k \leq 8$. On the other hand, for $D$ is a flag-transitive 6-design admitting $G \leq \operatorname{Aut}(D)$, we deduce from [[12], prop.3.6 (b)] the necessary condition that $2^{d}-3$ must divide $\left(\begin{array}{l}k \\ 4\end{array}\right)$, and hence it follows for each respective value of $k$ that $d=3$, contradicting our assumption.

Case (3): $G \cong A_{4}, v=2^{4}$

For $v=2^{4}$, we have $k \leq 9$, by Corollary 2.7. By Lemma 2.4 and Lemma 2.3, we have $k \neq 7,8,9$.

\subsection{Groups of Automorphisms of Almost Simple Type}

Case (1): $A_{v}, v \geq 5$

Since $D$ is non-trivial with $k>6$, we may assume that $v \geq 8$. Then $A_{v}$ is 6-transitive on $X$, and hence $G$ is $k$-transitive, this yields $D$ containing all of the $k$-subset of $X$. So $D$ is a trivial design, a contradiction.

Case (2): $\operatorname{PLS}(2, q), v=q+1, q=p^{e}>3$;
Here $N=P L S(2, q), v=q+1, q=p^{e} \geq 3$ and $p>3$, so $\operatorname{Aut}(N)=P \Gamma L(2, q),|G|=(q+1) q \frac{q-1}{d} a$ with $d=(2, q-1)$ and $a \mid d e$. We may again assume that $v=q+1 \geq 8$.

We will first assume that $N=G$. Then, by Remark 2.8, we obtain

$$
4(q-2)(q-3)(q-4)\left|P S L(2, q)_{x B}\right|=(k-1)(k-2)(k-3)(k-4)(k-5) .
$$

In view of Lemma 2.6, we have

$$
2(q-4) \geq(k-4)(k-5)
$$

It follows from Equation (1) that

$$
2(q-2)(q-3)\left|\operatorname{PSL}(2, q)_{x B}\right| \leq(k-1)(k-2)(k-3)
$$

If we assume that $k \geq 21$, then obviously

$$
2(k-1)(k-2)(k-3)<[(k-4)(k-5)]^{2}
$$

and hence

$$
(q-2)(q-3)\left|\operatorname{PSL}(2, q)_{x B}\right|<2(q-4)^{2}
$$

In view of inequality (2), clearly, this is only possible when $\left|P S L(2, q)_{x B}\right|=1$. In particular, $q$ has not to be even. But then the right-hand side of Equation (1) is always divisible by 16 but never the left-hand side, a contradiction. If $k<21$, then the few remaining possibilities for $k$ can easily be ruled out by hand using Equation (1), Inequality (2), and Corollary 2.9.

Now, let us assume that $N<G \leq \operatorname{Aut}(N)$. We recall that $q=p^{e} \geq 7$, and will distinguish in the following the case $p>3, p=2$, and $p=3$.

First, let $p>3$. We define $G^{*}=G \cap\left(\operatorname{PSL}(2, q):\left\langle\tau_{\alpha}\right\rangle\right)$ with $\tau_{\alpha} \in \operatorname{Sym}\left(G F\left(p^{e}\right)\right) \cup\{\infty\} \cong S_{v}$ of order $e$ induced by the Frobenius automorphism $\alpha: G F\left(p^{e}\right) \rightarrow G F\left(p^{e}\right), x \mapsto x^{p}$. Then, by Dedekind's law, we can write

$$
G^{*}=\operatorname{PSL}(2, q):\left(G^{*} \cap\left\langle\tau_{\alpha}\right\rangle\right)
$$

Defining $P \Sigma L(2, q)=P S L(2, q):\left\langle\tau_{\alpha}\right\rangle$, it can easily be calculated that $P \Sigma L(2, q)_{0,1, \infty}=\left\langle\tau_{\alpha}\right\rangle$, and $\left\langle\tau_{\alpha}\right\rangle$ has precisely $p+1$ distinct fixed points (cf. e.g., [[13] Ch. 6.4, Lemma 2]). As $p>3$, we have therefore that $G_{0 B_{1}}^{*} \cap\left\langle\tau_{\alpha}\right\rangle \leq G^{*} \cap\left\langle\tau_{\alpha}\right\rangle \leq G_{F}^{*}$ for a flag $F=\left\{\left(0, B_{1}\right),\left(0, B^{\prime}\right)\right\}$ fixed with $\left\langle\tau_{\alpha}\right\rangle$ by the definition of $6-(v, k, 2)$ designs. On the other hand, every element of $G^{*} \cap\left\langle\tau_{\alpha}\right\rangle$ either fixes block $B_{1}$, or commute block $B_{1}$ with block $B^{\prime}$, thus the index $\left[G_{0 B_{1}}^{*} \cap\left\langle\tau_{\alpha}\right\rangle: G^{*} \cap\left\langle\tau_{\alpha}\right\rangle\right] \leq 2$. Clearly $\operatorname{PSL}(2, q) \cap\left(G^{*} \cap\left\langle\tau_{\alpha}\right\rangle\right)=1$.

Hence, we have 


$$
\begin{aligned}
\left|\left(0, B_{1}\right)^{G^{*}}\right| & =\left[G^{*}: G_{0 B_{1}}^{*}\right] \leq\left[\operatorname{PSL}(2, q) \cap\left(G^{*} \cap\left\langle\tau_{\alpha}\right\rangle\right): \operatorname{PSL}(2, q)_{0 B_{1}} \cap\left(G_{0 B_{1}}^{*} \cap\left\langle\tau_{\alpha}\right\rangle\right)\right] \\
& =c\left[\operatorname{PSL}(2, q): \operatorname{PSL}(2, q)_{0 B_{1}}\right]=c\left|\left(0, B_{1}\right)^{\operatorname{PSL}(2, q)}\right| .
\end{aligned}
$$

where $c=1$ or 2 . Thus, if we assume that $G^{*} \leq \operatorname{Aut}(D)$ acts already flag-transitively on $D$, then we obtain $b k=\left|\left(0, B_{1}\right)^{G^{*}}\right| \leq c\left|\left(0, B_{1}\right)^{P S L(2, q)}\right|$. Then either $b k=\left|\left(0, B_{1}\right)^{P S L(2, q)}\right|$, and $\operatorname{PSL}(2, q)$ acts on $D$ flag-transitively, that is the case when $N=G$; or $b k=2\left|\left(0, B_{1}\right)^{P S L(2, q)}\right|$, and $\operatorname{PSL}(2, q)$ has exactly two orbits of equal length on the sets of flags. Then, proceeding similarly to the case $N=G$ for each orbit on the set of the flags, we have that

$$
2(q-2)(q-3)(q-4)\left|\operatorname{PSL}(2, q)_{0 B_{1}}\right|=(k-1)(k-2)(k-3)(k-4)(k-5)
$$

Using again

$$
2(q-4) \geq(k-4)(k-5)
$$

We obtain

$$
2(q-2)(q-3)\left|\operatorname{PSL}(2, q)_{0 B_{1}}\right| \leq(k-1)(k-2)(k-3)
$$

If we assume that $k \geq 21$, then again

$$
(k-1)(k-2)(k-3) \leq 2[(k-4)(k-5)]^{2}
$$

and thus

$$
4(q-2)(q-3)\left|\operatorname{PSL}(2, q)_{0 B_{1}}\right| \leq(q-4)^{2}
$$

but this is impossible. The few remaining possibilities for $k<21$ can again easily be ruled out by hand.

Now, let $p=2$, then, clearly $N=P S L(2, q)=P G L(2, q)$, and we have $\operatorname{Aut}(N)=P \Sigma L(2, q)$. If we assume that $\left\langle\tau_{\alpha}\right\rangle$ is the subgroup of $P \Sigma L(2, q)_{0 B_{1}}$ for a flag $\left(0, B_{1}\right) \in \mathrm{B}$, then we have $G^{*}=G=P \Sigma L(2, q)$ and as clearly $\operatorname{PSL}(2, q) \cap\left\langle\tau_{\alpha}\right\rangle=1$, we can apply Equation $(*)$. Thus, $\operatorname{PSL}(2, q)$ must also be flagtransitive, which has already been considered. Therefore, we assume that $\left\langle\tau_{\alpha}\right\rangle$ is not the subgroup of $P \Sigma L(2, q)_{0 B_{1}}$. Let $s>2$ be a prime divisor of $e=\left|\left\langle\tau_{\alpha}\right\rangle\right|$. As the normal subgroup $H:=\left(P \Sigma L(2, q)_{0,1, \infty}\right)^{s} \leq\left\langle\tau_{\alpha}\right\rangle$ of index $s$ has precisely $p^{s}+1$ distinct fix points, we have $G \cap H \leq G_{0 B_{1}}$ for a flag $F=\left\{\left(0, B_{1}\right),\left(0, B^{\prime}\right)\right\}$ fixed with $\left\langle\tau_{\alpha}\right\rangle$ by the definition of $6-(v, k, 2)$ designs. It can then be deduced that $e=s^{u}$ for some $u \in \underline{N}$. Since if we assume for $G=P \Sigma L(2, q)$ that there exists a further prime divisor $\bar{s}>2$ of $e$ with $\bar{s} \neq s$, then $\bar{H}:=\left(P \Sigma L(2, q)_{0,1, \infty}\right)^{\bar{s}} \leq\left\langle\tau_{\alpha}\right\rangle$ and $H$ are both subgroups of $P \Sigma L(2, q)_{0 B_{1}}$ by the flag-transitivity of $P \Sigma L(2, q)$, and hence $\left\langle\tau_{\alpha}\right\rangle \leq P \Sigma L(2, q)_{0 B_{1}}$, a contradiction. Furthermore, as $\left\langle\tau_{\alpha}\right\rangle$ is not the subgroup of $P \Sigma L(2, q)_{0 B_{1}}$. We may, by applying Dedekind's law, assume that

$$
G_{0 B_{1}}=P S L(2, q)_{0 B_{1}}:(G \cap H)
$$

Thus, by Remark 2.8, we obtain

$$
(q-2)(q-3)(q-4)\left|P S L(2, q)_{0 B_{1}}\right| G \cap H|=k(k-1)(k-2)(k-3)(k-4)(k-5)| G \cap\left\langle\tau_{\alpha}\right\rangle \mid
$$

More precisely:

$$
\begin{aligned}
\text { (A) if } G= & P S L(2, q):(G \cap H), \\
& (q-2)(q-3)(q-4)\left|\operatorname{PSL}(2, q)_{0 B_{1}}\right|=k(k-1)(k-2)(k-3)(k-4)(k-5)
\end{aligned}
$$

(B) if $G=P \Sigma L(2, q)$, 


$$
(q-2)(q-3)(q-4)\left|\operatorname{PSL}(2, q)_{0 B_{1}}\right|=k(k-1)(k-2)(k-3)(k-4)(k-5) s
$$

As far as condition (A) is concerned, we may argue exactly as in the earlier case $N=G$. Thus, only condition (B) remains. If $e$ is a power of 2, then Remark 2.8 gives

$$
(q-2)(q-3)(q-4)\left|G_{0 B_{1}}\right|=k(k-1)(k-2)(k-3)(k-4)(k-5) a
$$

with $a \mid e$. In particular, $a$ must divide $\left|G_{0 B_{1}}\right|$, and we may proceed similarly as in the case $N=G$, yielding a contradiction.

The case $p=3$ may be treated as the case $p=2$.

Case (3): $M_{v}, v=11,12,22,23,24$

By Corollary 2.7, we get $k=7$ for $v=11$ or 12 , and $k=7$ or 8 for $v=22,23$ or 24, and the very small number of cases for $k$ can easily be eliminated by hand using Corollary 2.9 and Remark 2.8 .

Case (4): $M_{11}, v=12$

As in case (3), for $v=12$, we have $k=7$ in view of Corollary 2.7, a contradiction since no 6-(12, 7, 2) design can exist by Corollary 2.9. This completes the proof of the Main Theorem.

\section{Acknowledgements}

The authors thank the referees for their valuable comments and suggestions on this paper.

\section{References}

[1] Kantor, W.M. (1985) Homogeneous Designs and Geometric Lattices. Journal of Combinatorial Theory, Series A, 38, 66-74. http://dx.doi.org/10.1016/0097-3165(85)90022-6

[2] Liebeck, M.W. (1993) 2-Transitive and Flag-Transitive Designs. In: Jungnickel, D. and Vanstone, S.A., Eds., Coding Theory, Design Theory, Group Theory, Wiley, New York, 13-30.

[3] Huber, M. (2004) The Classification of Flag-Transitive Steiner 3-Designs. Transactions of the American Mathematical Society, 1, 11-25.

[4] Huber, M. (2005) The Classification of Flag 2-Transitive Steiner 3-designs. Advances in Geometry, 5, 195-221. http://dx.doi.org/10.1515/advg.2005.5.2.195

[5] Cameron, P.J., Maimani, H.R. and Omidi, G.R. (2006) 3-Designs from PSL(2, q). Discrete Mathematics, 306, 30633073. http://dx.doi.org/10.1016/j.disc.2005.06.041

[6] Huber, M. (2007) The Classification of Flag-Transitive Steiner 4-Designs. Journal of Algebraic Combinatorics, 26, 183-207. http://dx.doi.org/10.1007/s10801-006-0053-0

[7] Huber, M. (2008) Steiner t-Designs for Large t. In: Calmet, J., Geiselmann, W., Mueller-Quade, J., Eds., Springer Lecture Notes in Computer Science, Springer, Berlin, Heidelberg, New York, 18-26.

[8] Cameron, P.J. and Praeger, C.E. (1992) Block-Transitive t-Designs. Finite Geometry and Combinatorics, 191, 103119.

[9] Huber, M. (2007) A Census of Highly Symmetric Combinatorial Designs. Journal of Algebraic Combinatorics, 26, 453-476. http://dx.doi.org/10.1007/s10801-007-0065-4

[10] Beth, T., Jungnickel, D., Lenz, H. (1999) Design Theory. Cambiridge University Press, Cambridge.

[11] Liu W.J., Tan, Q.H., Gong, L.Z. (2010) Flag-Transitive 5-(v, k, 2) Designs. Journal of Jiang-Su University (Natural Science Edition), 5, 612-615.

[12] Cameron, P.J. and Praeger, C.E. (1993) Block-Transitive t-Designs, II: Large t. In: De Clerck, F., et al., Eds., Finite Geometry and Combinatorics, London Mathematical Society Lecture Note Series No. 191, Cambridge University Press, Cambridge, 103-119.

[13] Dembowski, P. (1968) Finite Geometries. Springer, Berlin, Heidelberg, New York. 